\title{
Positive Herpes Simplex Virus Culture
}

National Cancer Institute

\section{Source}

National Cancer Institute. Positive Herpes Simplex Virus Culture. NCI Thesaurus. Code C122575.

Laboratory test results demonstrating the presence of the Herpes Simplex virus in a specimen. 